\title{
Long intergenic noncoding RNA p21 mediates oxidized LDL-induced apoptosis and expression of LOX-1 in human coronary artery endothelial cells
}

\author{
$\mathrm{TAO}_{\mathrm{ZHOU}^{1}}$ and XIAOFANG CHEN ${ }^{2}$ \\ ${ }^{1}$ Department of Cardiology; ${ }^{2}$ Nursing Department, The Third Affiliated Hospital, \\ South Medical University, Guangzhou, Guangdong 510630, P.R. China
}

Received November 24, 2015; Accepted November 15, 2016

DOI: $10.3892 / \mathrm{mmr} .2017 .7623$

\begin{abstract}
Atherosclerosis is the most common pathological cause of cardiovascular diseases, and endothelial dysfunction has a vital role. It has been suggested that inhibiting endothelial cell apoptosis induced by oxidized low-density lipoprotein (ox-LDL), an essential atherosclerotic factor, is a potential novel therapeutic strategy against atherosclerosis. Previous studies have revealed that endothelial lectin-like ox-LDL receptor-1 (LOX-1) and long intergenic noncoding RNA p21 (lincRNA-p21) may serve as therapeutic targets for atherosclerosis and associated cardiovascular disorders. The present study investigated the role of lincRNA-p21 in oxLDL-induced apoptosis and expression of LOX-1 in human coronary artery endothelial cells (HCAECs). Primary HCAECs were treated with ox-LDL (30, 60 or $90 \mu \mathrm{g} / \mathrm{ml})$ for 24 or $48 \mathrm{~h}$, and the expression of lincRNA-p21, LOX-1 and cell apoptosis rate were measured. Ox-LDL dose- and time-dependently induced the expression of lincRNA-p21 and LOX-1 and apoptosis in HCAECs. Lentiviral overexpression of lincRNA-p21 markedly increased oxLDL-induced apoptosis and the expression of LOX-1 in HCAECs. Additionally, the effect was largely blocked by selective protein kinase C (PKC) inhibitor, rottlerin. However, lentiviral knockdown of lincRNA-p21 markedly decreased oxLDL-induced apoptosis and the expression of LOX-1. In addition, overexpression and knockdown of lincRNA-p21 markedly increased and decreased oxLDL-induced PKC $\delta$ activity/phosphorylation, respectively. In conclusion, to the best of our knowledge, the present study provides the first evidence indicating that lincRNA-p21 is a major mediator of oxLDL-induced apoptosis and expression of LOX-1 in human vascular endothelial cells, and acts via
\end{abstract}

Correspondence to: Dr Xiaofang Chen, Nursing Department, The Third Affiliated Hospital, South Medical University, $183 \mathrm{~W}$ Zhongshan Avenue, Guangzhou, Guangdong 510630, P.R. China E-mail: chenxiaofang250@gmail.com

Key words: long intergenic noncoding RNA p21, oxidized LDL, lectin-like ox-LDL receptor-1, apoptosis, endothelial cells, atherosclerosis, protein kinase $\mathrm{C} \delta$ activation of PKCס. These results provide insights into the role of lincRNA-p21 in the pathogenesis of atherosclerosis.

\section{Introduction}

Atherosclerosis is the most common pathological cause of cardiovascular diseases (1), and endothelial dysfunction has a vital role in atherosclerosis (2). Endothelial cells form the inner lining of the blood vessels and regulate vascular function and homeostasis. Under pathological conditions, endothelial cell apoptosis functions as an initiating step for atherosclerosis, as apoptotic endothelial cells are pro-thrombotic and pro-proliferative and contribute to the ensuing atherogenic processes (3). Oxidized low-density lipoprotein (ox-LDL), an essential atherosclerotic risk factor, is reportedly to be crucial for multiple functional alternations during the pathogenesis of atherosclerosis, including inducing endothelial cell apoptosis $(4,5)$. The morphological changes of cultured endothelial cells exposed to ox-LDL are similar to those observed in the endothelium covering atherosclerotic lesions (6). Therefore, inhibiting endothelial cell apoptosis induced by ox-LDL is a potential novel therapeutic strategy against atherosclerosis.

Lectin-like ox-LDL receptor-1 (LOX-1), a small transmembrane glycoprotein of $50 \mathrm{kDa}$ encoded by the oxidized low-density lipoprotein receptor 1 gene (7), is a scavenger receptor originally identified as the primary receptor for ox-LDL uptake in endothelial cells (8). Activation of LOX-1 by ox-LDL mediates pro-atherogenic cellular responses implicated in the pathogenesis of atherosclerosis, including endothelial dysfunction characterized by reduced endothelium-dependent relaxation, increased monocyte adhesion to endothelial cells, and endothelial cell apoptosis and senescence (8). Basal LOX-1 expression in endothelial cell is low, however it is induced by various stimuli associated with atherosclerosis, including pro-inflammatory cytokines and ox-LDL (9). In human atherosclerotic lesions, LOX-1 is overexpressed in endothelial cells (9). Therefore, LOX-1 represents an attractive therapeutic target for the treatment of human atherosclerotic diseases.

A novel class of non-coding RNAs, longer than 200 nucleotides and termed long noncoding RNAs (IncRNAs), reportedly are involved in the regulation of gene expression 
through epigenetic mechanisms, including chromatin remodeling, regulation of splicing and by acting as sponges for microRNAs $(10,11)$. Previous studies have revealed that lncRNAs are important in vascular injury and remodeling, which are the leading causes of cardiovascular diseases (12). Long intergenic noncoding RNA p21 (lincRNA-p21) is a downstream lncRNA transcript of $\mathrm{p} 53$. It has been previously reported that lincRNA-p21 acts via several mechanisms ranging from repressing genes in the p53 transcriptional network to regulating mRNA translation and protein stability, and participates in diverse biological processes, including apoptosis (13). Recent studies have demonstrated that lincRNA-p21 inhibits proliferation and promotes apoptosis in vascular endothelial cells and smooth muscle cells, suggesting that this lncRNA may be useful as a therapeutic target for atherosclerosis and associated cardiovascular disorders $(14,15)$.

The present study aimed to investigate the role of lincRNA-p21 in oxLDL-induced apoptosis and expression of LOX-1 in human coronary artery endothelial cells (HCAECs).

\section{Materials and methods}

Cell culture. Primary HCAECs (cat. no. 300-05a) and MesoEndo cell growth medium (cat. no. 212-500) were purchased from Cell Applications, Inc. (San Diego, CA, USA). HCAECs were cultured in MesoEndo cell growth medium supplemented with $5 \%$ fetal bovine serum (Thermo Fisher Scientific, Inc., Waltham, MA, USA) and 100 U/ml penicillin-streptomycin (Sigma-Aldrich; Merck Millipore, Darmstadt, Germany) in an incubator with a humidified atmosphere of $95 \%$ air and $5 \% \mathrm{CO}_{2}$ at $37^{\circ} \mathrm{C}$.

Preparation of ox-LDL and cell treatment. Native LDL (density, 1.019-1.063 g/ml) was separated from the fresh normolipidemic human serum purchased from Guangdong Blood Bank (Guangzhou, China) by discontinuous density-gradient ultracentrifugation as previously described (16). Isolated LDL was desalted using an Econo-Pac 10 DG chromatography column (Bio-Rad Laboratories, Inc., Hercules, CA, USA) and sterile-filtered $(0.22 \mu \mathrm{m}$ pore size; EMD Millipore, Billerica, MA, USA). To oxidize LDL, the lipoprotein $(0.5 \mathrm{mg} / \mathrm{ml}$ in sterile PBS) was incubated with $5 \mu \mathrm{M} \mathrm{CuSO}_{4}$ at $37^{\circ} \mathrm{C}$ for $20 \mathrm{~h}$. Oxidized LDL was concentrated by centrifuging in Amicon Centriplus YM-100 tubes (EMD Millipore) for $2 \mathrm{~h}$ at 3,000 x $g$ and $8^{\circ} \mathrm{C}$, and subsequently sterile-filtered. The oxidation was confirmed by measuring thiobarbituric acid-reactive substances using tetraethoxypropane as a standard (17). HCAECs were treated with ox-LDL $(30,60$ or $90 \mu \mathrm{g} / \mathrm{ml})$ for 24 or $48 \mathrm{~h}$ with selective protein kinase $\mathrm{C}$ (PKC) $\delta$ inhibitor, rottlerin $(1 \mu \mathrm{M}$; cat. no. CAS 82-08-6; Santa Cruz Biotechnology, Inc., Dallas, TX, USA) (18). Untreated HCAECs $(0 \mu \mathrm{g} / \mathrm{ml}$ ox-LDL) were used as controls.

Lentiviral transduction. Total RNA from cultured HCAECs was isolated using miRNeasy kits (Qiagen China Co., Ltd., Shanghai, China) according to the manufacturer's protocol, followed by purification with TURBO DNA-free System (Ambion; Thermo Fisher Scientific Inc.). RT was performed on $1,000 \mathrm{ng}$ total RNA using MulV reverse transcriptase (Thermo Fisher Scientific, Inc.) and random hexamer primers (Thermo
Fisher Scientific, Inc.) in a $20-\mu 1$ reaction [ $2 \mu 1$ RNA (500 ng/ $\mu \mathrm{l})$, $1 \mu \mathrm{l}$ MulV reverse transcriptase $(50 \mathrm{U} / \mu \mathrm{l}), 1 \mu \mathrm{l}$ hexamer primers (100 $\mu \mathrm{M}), 2 \mu \mathrm{l}$ 10X RT reaction buffer, and $14 \mu \mathrm{l}$ RNAse-free water] and incubated at $42^{\circ} \mathrm{C}$ for $1 \mathrm{~h}$. Total RNA from cultured HCAECs was isolated using miRNeasy kits (Qiagen China Co., Ltd.) according to the manufacturer's protocol, followed by purification with TURBO DNA-free System (Ambion; Thermo Fisher Scientific Inc.). The lincRNA-p21 cDNA was amplified by PCR using the extracted total RNA and cloning primers with sequences as follows: 5'-TGGCAGTCT GACCCACACTCCCCACGCCC-3' (forward) and 5'-ACA GTGCACAGACAATCATACACACGTGT-3' (reverse). Lentivirus expressing human lincRNA-p21 was generated by sub-cloning the above amplified human lincRNA-p21 cDNA to the pLenti-GIII-CMV lentivirus expression system (Applied Biological Materials, Inc., Richmond, BC, Canada). The lincRNA-p21 lentiviral vector or blank control lentivector was transfected with the packaging vectors psPAX2 (Addgene Inc., Cambridge, MA, USA) and pMD2.G (Addegene Inc.) into 293T cells (American Type Culture Collection, Manassas, VA, USA) by calcium chloride to produce the lentivirus, which was subsequently used to transduce HCAECs. Human lincRNA-p21-small interfering RNA (siRNA) and scrambled control siRNA lentivirus particles were designed and ordered from GenePharma Co., Ltd. (Shanghai, China). The siRNA sequence targeting lincRNA-p21 was as follows: 5'-GGAGGA CACAGGAGAGGCA-3'. The scrambled control sequence was as follows: 5'-GGCGCAGAGACGGAGAGAA-3'. At $6 \mathrm{~h}$ after lentiviral transduction, HCAECs were subject to ox-LDL treatment.

Reverse transcription-quantitative polymerase chain reaction (RT-qPCR). Total RNA from cultured HCAECs was isolated using miRNeasy kits (Qiagen China Co., Ltd.) according to the manufacturer's protocol, followed by purification with TURBO DNA-free System (Ambion; Thermo Fisher Scientific, Inc.). For measuring lncRNAs, RT was performed on 1,000 ng total RNA using MulV reverse transcriptase (Thermo Fisher Scientific, Inc.) and random hexamer primers (Thermo Fisher Scientific, Inc.) in a $20-\mu 1$ reaction [ $2 \mu \mathrm{l}$ RNA (500 ng/ $\mu \mathrm{l}), 1 \mu \mathrm{l}$ MulV reverse transcriptase $(50 \mathrm{U} / \mu \mathrm{l}), 1 \mu \mathrm{l}$ hexamer primers $(100 \mu \mathrm{M}), 2 \mu \mathrm{l} 10 \mathrm{X}$ RT reaction buffer, and $14 \mu \mathrm{l}$ RNAse-free water] and incubated at $42^{\circ} \mathrm{C}$ for $1 \mathrm{~h}$. cDNA was used as template for qPCR using an ABI-Prism 7700 Sequence Detection system and the Fast SYBR Green (Applied Biosystems; Thermo Fisher Scientific, Inc.). Human ribosomal P0 (RPLP0) mRNA was as the reference gene. Primer sequences used are as follows: LincRNA-p21, 5'-CCTGTCCCACTCGCTTTC-3' (forward) and 5'-GGAACTGGAGACGGAATGTC-3' (reverse); LOX-1, 5'-TTACTCTCCATGGTGGTGGTGCC-3' (forward) and 5'-AGCTTCTTCTGCTTGTTGCC-3-3' (reverse); RPLP0, 5'-TCGACAATGGCAGCATCTAC-3' (forward) and 5'-ATC CGTCTCCACAGACAAGG-3' (reverse). Analysis of relative gene expression levels was performed using the formula $2^{-\Delta \mathrm{Cq}}$, with $\Delta \mathrm{Cq}=\mathrm{Cq}_{\text {(target gene) }}-\mathrm{Cq}_{\text {(control) }}$ (19).

Cell apoptosis assay. HCAECs were cultured at $5 \times 10^{4}$ cells per well in 96-well tissue culture plates and subject to ox-LDL treatment $(30,60$ or $90 \mu \mathrm{g} / \mathrm{ml})$ for 24 or $48 \mathrm{~h}$. Cell apoptosis was measured at 24 or $48 \mathrm{~h}$ of ox-LDL treatment with a 
A

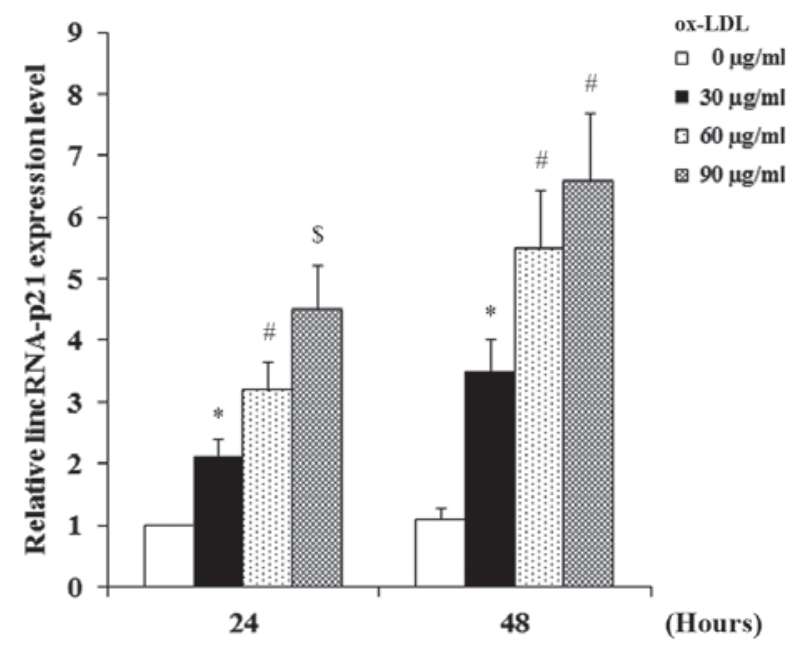

B

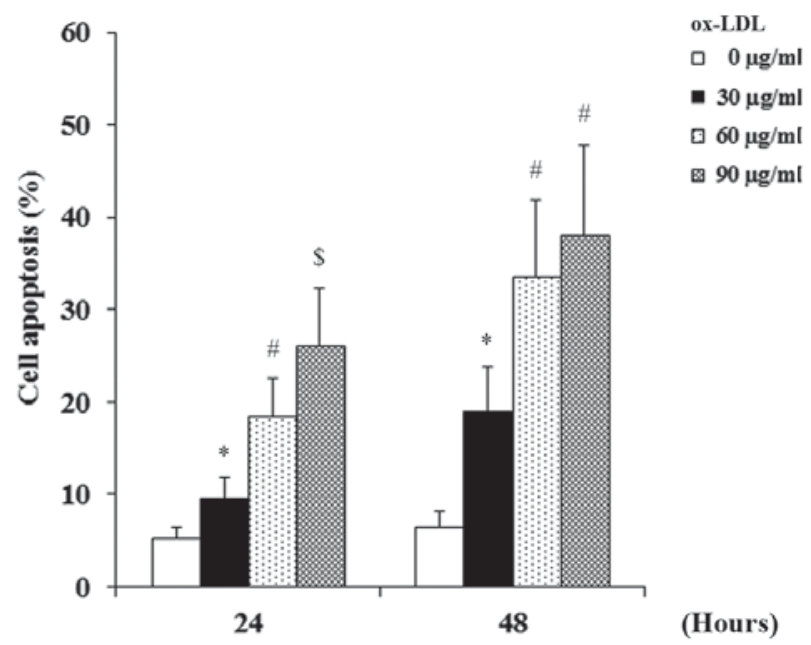

Figure 1. Expression of lincRNA-p21 and apoptosis in HCAECs under ox-LDL treatment. HCAECs were treated with ox-LDL (30, 60 or $90 \mu$ g/ml) for 24 or $48 \mathrm{~h}$. HCAECs under normal culture conditions $(0 \mu \mathrm{g} / \mathrm{ml}$ ox-LDL treatment) were used as parallel controls. (A) The expression of lincRNA-p21 was measured at 24 and $48 \mathrm{~h}$ of ox-LDL treatment with reverse transcription-quantitative polymerase chain reaction and expressed as fold-changes to that of HCAECs under normal culture conditions $(0 \mu \mathrm{g} / \mathrm{ml}$ ox-LDL treatment) for $24 \mathrm{~h}$ (designated as 1$)$. (B) The apoptosis rate of HCAECs was measured at 24 and $48 \mathrm{~h}$ ox-LDL treatment with a microplate reader-based apoptosis detection kit. ${ }^{*} \mathrm{P}<0.05 \mathrm{vs} .0 \mu \mathrm{g} / \mathrm{ml} ;{ }^{\#} \mathrm{P}<0.05 \mathrm{vs} .30 \mu \mathrm{g} / \mathrm{ml} ;{ }^{\$} \mathrm{P}<0.05 \mathrm{vs} .60 \mu \mathrm{g} / \mathrm{ml}$. HCAECs, human coronary artery endothelial cells; ox-LDL, oxidized low-density lipoprotein; lincRNA-p21, long intergenic noncoding RNA p21.

microplate reader-based TiterTACS in situ apoptosis detection kit (cat. no. 4822-96-K; R\&D Systems, Inc., Minneapolis, MN, USA) as described by the manufacturer. Each experiment was repeated three times in triplicates.

Western blot analysis. Cells was lysed with a hypotonic buffer containing $2 \%$ Nonidet-P and a protease inhibitor cocktail (Sigma-Aldrich; Merck Millipore) by sonication three times for $3 \mathrm{sec}$ on ice. The supernatant obtained after centrifugation at $2,000 \times \mathrm{g}$ for $15 \mathrm{~min}$ at $4^{\circ} \mathrm{C}$ was used for protein concentration determination by the Coomassie blue method (Thermo Fisher Scientific,Inc.) and for subsequent steps. Equal amount of proteins (5 $\mu \mathrm{g})$ for each sample were separated by 10\% SDS-PAGE and blotted onto a polyvinylidene difluoride microporous membrane (EMD Millipore). The membranes were blocked with 5\% skim milk powder in TBS-Tween $(0.1 \%)$ for $2 \mathrm{~h}$ and incubated for $1 \mathrm{~h}$ with a 1:1,000 dilution of goat anti-human LOX-1 polyclonal antibody (cat. no. sc-11653; Santa Cruz Biotechnology, Inc.), mouse anti-human $\beta$-actin monoclonal antibody (cat. no. sc-81178; Santa Cruz Biotechnology, Inc.), goat anti-human

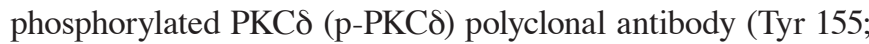
cat. no. sc-18367; Santa Cruz Biotechnology, Inc.), or rabbit anti-human PKCס polyclonal antibody (cat. no. sc-937; Santa Cruz Biotechnology, Inc.). The membranes were then washed with TBS-Tween $(0.1 \%)$ and probed using bovine anti-goat (cat. no. sc-2350; Santa Cruz Biotechnology, Inc.), anti-rabbit (cat. no. sc-2370; Santa Cruz Biotechnology, Inc.) or anti-mouse (cat. no. sc-2371; Santa Cruz Biotechnology, Inc.) secondary antibody (1:5,000 dilution for $1 \mathrm{~h})$ conjugated to horseradish peroxidase. Peroxidase was detected with a GE Healthcare enhanced chemiluminescence kit (GE Healthcare Life Sciences, Shanghai, China) using the ChemiDoc Touch Imaging System (Bio-Rad Laboratories Inc.) and Image Lab ${ }^{\mathrm{TM}}$ Touch Software (Bio-Rad Laboratories Inc.) for image acquisition and onboard image analysis. Three independent experiments were performed.
PKC $\delta$ activity assay. PKC $\delta$ activity assays were performed as previously described (20). Briefly,HCAEClysate was pre-cleaned with protein A/G plus-agarose (Santa Cruz Biotechnology, Inc.) and then incubated with rabbit anti-human PKC $\delta$ polyclonal antibody (cat. no. sc-937; Santa Cruz Biotechnology, Inc.) overnight at $4^{\circ} \mathrm{C}$. PKC $\delta$ immunocomplexes were then pulled down by $3 \mathrm{~h}$ of incubation with $30 \mu \mathrm{l}$ protein $\mathrm{A} / \mathrm{G}$ plus-agarose. The immunocomplexes were resuspended in $2 \mathrm{X}$ kinase reaction buffer $\left[300 \mathrm{mM} \mathrm{NaCl}, 8 \mathrm{mM} \mathrm{MnCl}{ }_{2}, 12 \mathrm{mM} \mathrm{MgCl}_{2}, 20 \%\right.$ (vol/vol) glycerol, $20 \mu \mathrm{M}$ ATP, $2 \mathrm{mM}$ dithiothreitol, $200 \mu \mathrm{M}$ $\mathrm{Na}_{3} \mathrm{VO}_{4}$, and $100 \mathrm{mM}$ Hepes ( $\mathrm{pH}$ 7.5)]. The protein kinase reaction was initiated by adding $5 \mu \mathrm{Ci}\left[\gamma^{32} \mathrm{P}\right]$ ATP (GE Healthcare Life Sciences) and $100 \mathrm{ng}$ histone mixture extracted from calf thymus (Sigma-Aldrich; Merck Millipore) into $20 \mu \mathrm{l}$ reaction buffer containing immunoprecipitated PKC . After $20 \mathrm{~min}$ of incubation at $30^{\circ} \mathrm{C}$, the reaction was terminated by adding $2 \%$ SDS gel loading buffer. The samples were then subjected to gel electrophoresis, and phosphorylated histone was revealed by autoradiography to indicate PKC $\delta$ kinase activity.

Statistical analysis. Statistical analyses were performed using SPSS for Windows 19.0 (IBM SPSS, Armonk, NY, USA). All values are expressed as the mean \pm standard deviation. Comparisons of means among multiple groups were performed with one-way analysis of variance followed by post hoc pairwise comparisons using Tukey's tests. Two-tailed $\mathrm{P}<0.05$ was considered to indicate a statistically significant difference.

\section{Results}

$O x$-LDL induces expression of lincRNA-p21 and LOX-1, and induces apoptosis in HCAECs. HCAECs were treated with ox-LDL (30, 60 or $90 \mu \mathrm{g} / \mathrm{ml})$ for 24 or $48 \mathrm{~h}$. As demonstrated in Fig. 1, ox-LDL dose- and time-dependently induced the expression of lincRNA-p21 (Fig. 1A) and apoptosis (Fig. 1B) in 
HCAECs. As demonstrated in Fig. 2, the constitutive expression level of LOX-1 in HCAECs was low; ox-LDL dose- and time-dependently induced the mRNA (Fig. 2A) and protein (Fig. 2B) expression of LOX-1 in HCAECs. The similar trends of oxLDL-induced expression of lincRNA-p21 and LOX-1, and apoptosis in HCAECs suggested that lincRNA-p21 may be associated with oxLDL-induced expression of LOX-1 and apoptosis in HCAECs.

Effect of lincRNA-p21 on oxLDL-induced apoptosis and expression of LOX-1 in HCAECs. Subsequently, HCAECs were transduced with human lincRNA-p21 lentivirus or human lincRNA-p21 siRNA lentivirus to overexpress or knockdown lincRNA-p21, respectively. HCAECs without lentiviral transduction and those transduced with the blank control lentivirus or a scrambled siRNA lentivirus were used as controls. The cells in all groups were treated with $90 \mu \mathrm{g} / \mathrm{ml}$ ox-LDL for $48 \mathrm{~h}$ with or without $1 \mu \mathrm{M}$ selective PKC $\delta$ inhibitor, rottlerin (18). As presented in Fig. 3A, lentiviral transduction of lincRNA-p21 and lincRNA-p21-siRNA resulted in significant increase and decrease of the expression of lincRNA-p21, respectively compared with the control groups $(\mathrm{P}=0.0032)$. Inhibition of PKC $\delta$ by rottlerin exhibited no significant effect on the expression of lincRNA-p21 compared with the control groups. As demonstrated in Fig. 3B, overexpression of lincRNA-p21 significantly enhanced oxLDL-induced apoptosis from $\sim 40 \%$ in the controls to $\sim 93 \%$ ( $\mathrm{P}=0.0007)$. Inhibition of $\mathrm{PKC} \delta$ signaling by rottlerin combined with lincRNA-21 overexpression decreased the rate back to $\sim 60 \%$. However, knockdown of lincRNA-p21 significantly decreased oxLDL-induced apoptosis from $\sim 40 \%$ in the control group to $\sim 14 \%$ ( $\mathrm{P}=0.0014$; Fig. 3B).

In HCAECs treated with $90 \mu \mathrm{g} / \mathrm{ml}$ ox-LDL for $48 \mathrm{~h}$, the mRNA and protein expression of LOX-1 was measured and expressed as fold-changes to that of HCAECs under normal culture conditions $(0 \mu \mathrm{g} / \mathrm{ml}$ ox-LDL treatment) for $24 \mathrm{~h}$ (designated as 1). As demonstrated in Fig. 4, ox-LDL significantly induced the expression of LOX-1 in HCAECs, as the control groups exhibited a $>4$-fold increase in expression of LOX-1 at both the mRNA (Fig. 4A) and the protein (Fig. 4B) levels. Compared with the controls, overexpression of lincRNA-p21 significantly enhanced the oxLDL-induced expression of LOX-1 $(\mathrm{P}=0.0025)$. The enhancing effect of lincRNA-p21 on LOX-1 expression at the mRNA and the protein levels was blocked by $\sim 57 \%$ by rottlerin (Fig. 4). However, knockdown of lincRNA-p21 significantly decreased oxLDL-induced expression of LOX-1 at the mRNA and the protein levels by $~ 72 \%$ compared with the control groups ( $\mathrm{P}=0.0006$; Fig. 4). Rottlerin alone blocked $\sim 40 \%$ of oxLDL-induced apoptosis, suggesting that PKC $\delta$ is important in oxLDL-induced endothelial apoptosis. Taken together, the findings suggested that ox-LDL induced apoptosis and the expression of LOX-1 in HCAECs partially through lincRNA-p21 by a PKC $\delta$-dependent mechanism.

Effect of lincRNA-p21 on PKC $\delta$ activity and phosphorylation in HCAECs under ox-LDL treatment. In HCAECs treated with $90 \mu \mathrm{g} / \mathrm{ml}$ ox-LDL for $48 \mathrm{~h}$, the PKCd activity was measured and expressed as fold-changes to that of HCAECs under normal culture conditions ( $0 \mu \mathrm{g} / \mathrm{ml}$ ox-LDL treatment $)$
A

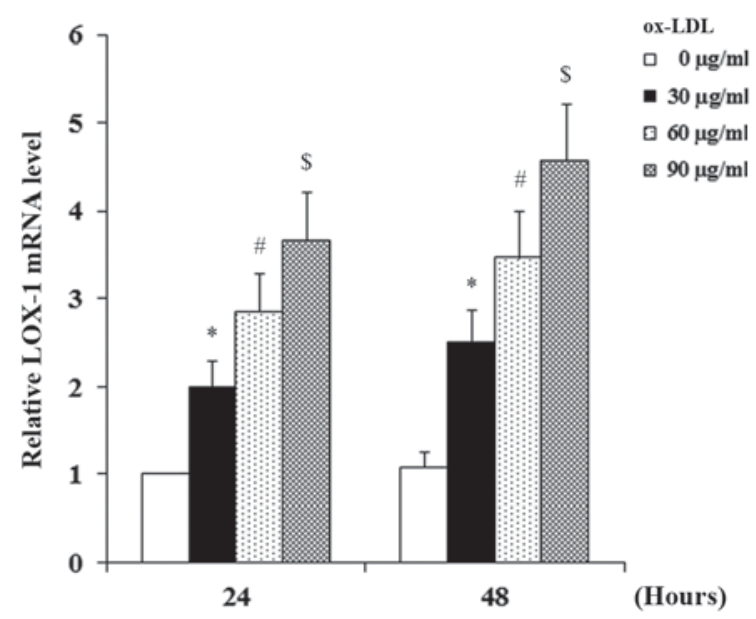

B
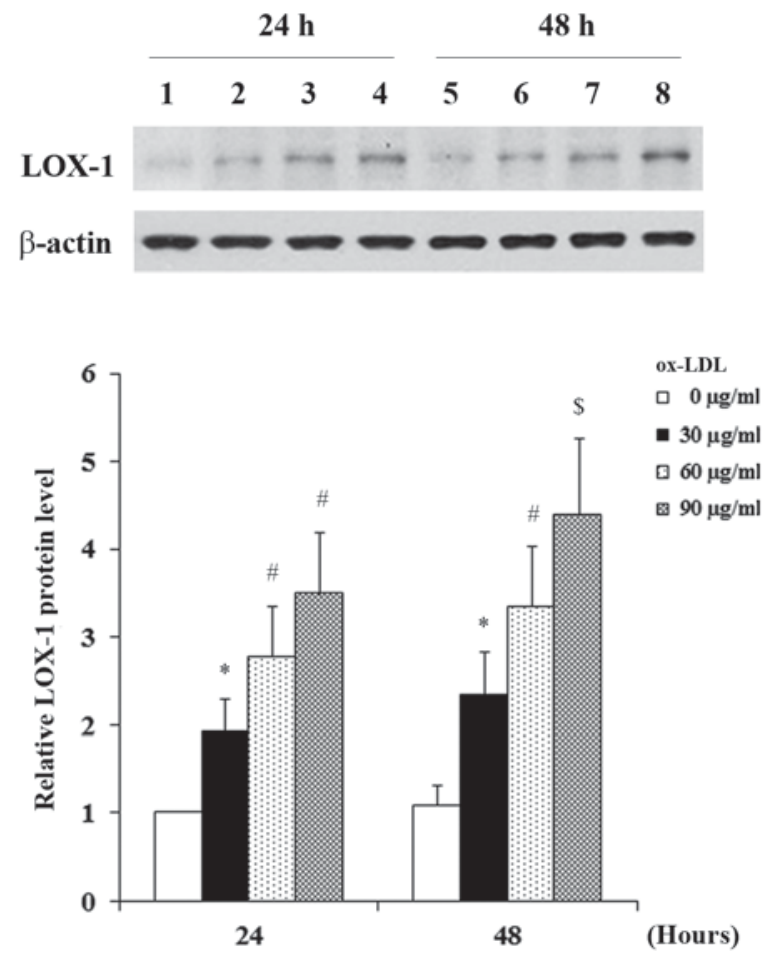

Figure 2. Expression of LOX-1 in HCAECs under ox-LDL treatment. HCAECs were treated with ox-LDL (30, 60 or $90 \mu \mathrm{g} / \mathrm{ml})$ for 24 or $48 \mathrm{~h}$. HCAECs under normal culture conditions $(0 \mu \mathrm{g} / \mathrm{ml}$ ox-LDL treatment $)$ were used as parallel controls. (A) LOX-1 mRNA levels were measured at 24 and 48 h of ox-LDL treatment with reverse transcription-quantitative polymerase chain reaction and expressed as fold-changes to that of HCAECs under normal culture conditions $(0 \mu \mathrm{g} / \mathrm{ml}$ ox-LDL treatment $)$ for $24 \mathrm{~h}$ (designated as 1). (B) LOX-1 protein levels were measured at 24 and $48 \mathrm{~h}$ of ox-LDL treatment via western blot analyses: Lanes 1 and 5, $0 \mu \mathrm{g} / \mathrm{ml}$ ox-LDL treatment; lanes 2 and 6, $30 \mu \mathrm{g} / \mathrm{ml}$ ox-LDL treatment; lanes 3 and 7 , $60 \mu \mathrm{g} / \mathrm{ml}$ ox-LDL treatment; and lanes 4 and $8,90 \mu \mathrm{g} / \mathrm{ml}$ ox-LDL treatment. $\beta$-actin blotting was used as a loading control. Density of the LOX-1 blot was normalized against that of the $\beta$-actin blot to obtain a relative blot density, which was expressed fold-changes to that of HCAECs under normal culture conditions $(0 \mu \mathrm{g} / \mathrm{ml}$ ox-LDL treatment) for $24 \mathrm{~h}$ (designated as 1$)$. ${ }^{*} \mathrm{P}<0.05$ vs. $0 \mu \mathrm{g} / \mathrm{ml}$; ${ }^{\#} \mathrm{P}<0.05$ vs. $30 \mu \mathrm{g} / \mathrm{ml}$; ${ }^{\$} \mathrm{P}<0.05$ vs. $60 \mu \mathrm{g} / \mathrm{ml}$. HCAECs, human coronary artery endothelial cells; ox-LDL, oxidized low-density lipoprotein; LOX-1, endothelial lectin-like ox-LDL receptor-1.

for $24 \mathrm{~h}$ (designated as 1). As demonstrate in Fig. 5A, ox-LDL significantly induced the PKC $\delta$ activity in HCAECs, as the 
A

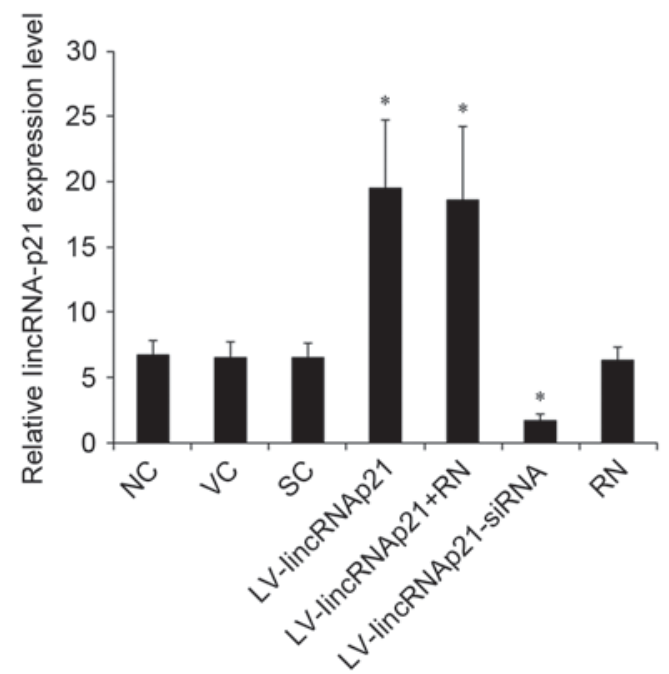

B

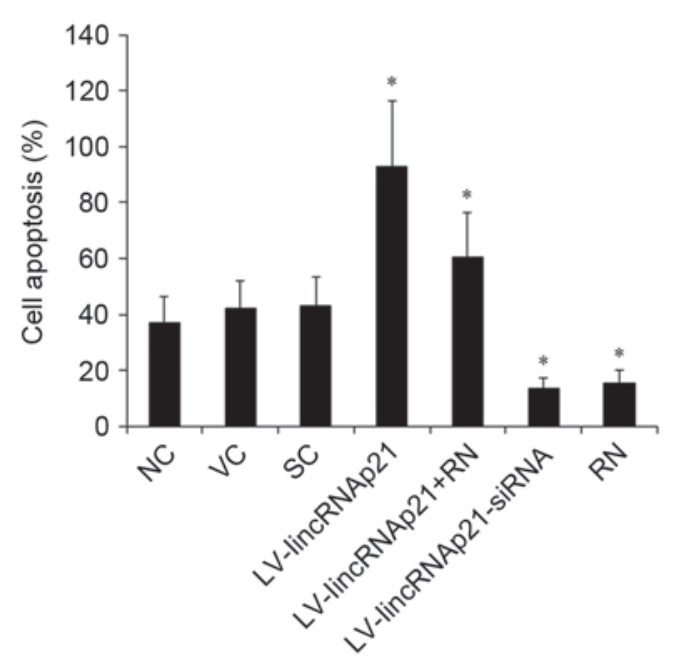

Figure 3. Effect of linkRNA-p21 on apoptosis in HCAECs under ox-LDL treatment. HCAECs were transduced with LV-lincRNA-p21 or LV-lincRNA-p21-siRNA to overexpress or knockdown lincRNA-p21, respectively. NC, VC and SC were used as controls. The cells were treated with $90 \mu \mathrm{g} / \mathrm{ml}$ ox-LDL for $48 \mathrm{~h}$ with or without $1 \mu \mathrm{M}$ selective protein kinase C- $\delta$ inhibitor, RN. (A) The expression of lincRNA-p21 was measured with reverse transcription-quantitative polymerase chain reaction and expressed as fold-changes to that of HCAECs under normal culture conditions ( $0 \mu \mathrm{g} / \mathrm{m}$ ox-LDL treatment) for $24 \mathrm{~h}$ (designated as 1$)$ (B) The apoptosis rate of HCAECs was measured with a microplate reader-based apoptosis detection kit. "P<0.05 vs. NC, VC and SC. HCAECs, human coronary artery endothelial cells; ox-LDL, oxidized low-density lipoprotein; LV, lentivirus; NC, no LV transduction; VC, blank control lentivirus; SC, scramble control lentivirus; lincRNA-p21, long intergenic noncoding RNA p21; RN, Rottlerin; siRNA, small interfering RNA.

control groups exhibited a $\sim 3.65$-fold increase in PKC $\delta$ activity compared with $0 \mu \mathrm{g} / \mathrm{ml}$ ox-LDL. Compared with the controls, overexpression of lincRNA-p21 significantly enhanced the oxLDL-induced PKC $\delta$ activity $(\mathrm{P}<0.05)$. The enhancing effect of lincRNA-p21 and ox-LDL was completely blocked by rottlerin (Fig. 5A). However, knockdown of lincRNA-p21 significantly decreased oxLDL-induced PKC 8 activity by $\sim 69 \%$ compared with the control groups (Fig. 5A). A similar data trend was observed with the PKC $\delta$ phosphorylation at Tyr 155 (Fig. 5B) (21,22). The findings indicated that PKCס is a downstream effector of lincRNA-p21 in HCAECs.

\section{Discussion}

It has been previous suggested that inhibiting oxLDL-induced endothelial cell apoptosis is a potential novel therapeutic strategy against atherosclerosis (23). Recent studies have revealed that endothelial LOX-1 and lincRNA-p21 may serve as therapeutic targets for atherosclerosis and associated cardiovascular disorders $(8,14,15)$. To the best of our knowledge, the present study provides the first evidence suggesting that lincRNA-p21 mediates oxLDL-induced apoptosis and expression of LOX-1 in human vascular endothelial cells, using HCAECs as an in vitro cell model.

In support of previous studies (9), the present study demonstrated that ox-LDL induced increased expression of LOX-1 and apoptosis in HCAECs. It was also demonstrated that ox-LDL induced increased expression of lincRNA-p21 in parallel, suggesting that lincRNA-p21 may be associated with oxLDL-induced expression of LOX-1 and apoptosis in HCAECs. Lentiviral overexpression and knockdown of lincRNA-p21 markedly increased and decreased oxLDL-induced apoptosis and the expression of LOX-1, respectively, suggesting that lincRNA-p21 is a major mediator of the effects of ox-LDL on the expression of LOX-1 and apoptosis in human vascular endothelial cells.

LOX-1 binds and internalizes ox-LDL by receptor-mediated endocytosis, which is the initial step that leads to oxLDL-induced apoptosis (7). In the current study, ox-LDL induced the expression of lincRNA-p21, which enhanced oxLDL-induced expression of LOX-1. The findings suggest that the oxLDL/LOX-1/lincRNA-p21 signaling may form a positive feedback loop, which facilitates the effect of ox-LDL on human vascular endothelial cells as manifested by enhanced apoptosis of HCAECs under ox-LDL treatment. Notably, although rottlerin, a selective PKC $\delta$ inhibitor, did not induce a significant effect on the expression of lincRNA-p21, it largely blocked the enhancing effect of lincRNA-p21 on oxLDL-induced expression of LOX-1 and apoptosis, suggesting that lincRNA-p21 mediates the effects of ox-LDL on human vascular endothelial cells partially via a $\mathrm{PKC} \delta$-dependent mechanism. This was subsequently corroborated by PKC $\delta$ activity assay results and the activation phosphorylation levels of PKC 8 , confirming that PKC $\delta$ is a downstream effector of lincRNA-p21 in HCAECs.

It has been previously reported that lincRNA-p21 acts via several mechanisms, ranging from repressing genes in the p53 transcriptional network, to regulating mRNA translation and protein stability (13). Wu et al (15) demonstrated that lincRNA-p21, a transcriptional target of p53, regulates vascular smooth muscle cell apoptosis and atherosclerosis by feeding back to enhance p53 transcriptional activity. Previous studies also have reported that $\mathrm{PKC} \delta$ mediates activation of p53 and promotes cell apoptosis (24-26). The findings of the current study suggest that lincRNA-p21 activates PKC $\delta$. This may enhance oxLDL-induced HCAEC apoptosis by: i) Increasing oxLDL-induced expression of LOX-1 and facilitating the positive feedback loop of oxLDL/LOX-1/lincRNA-p21 signaling; 
A

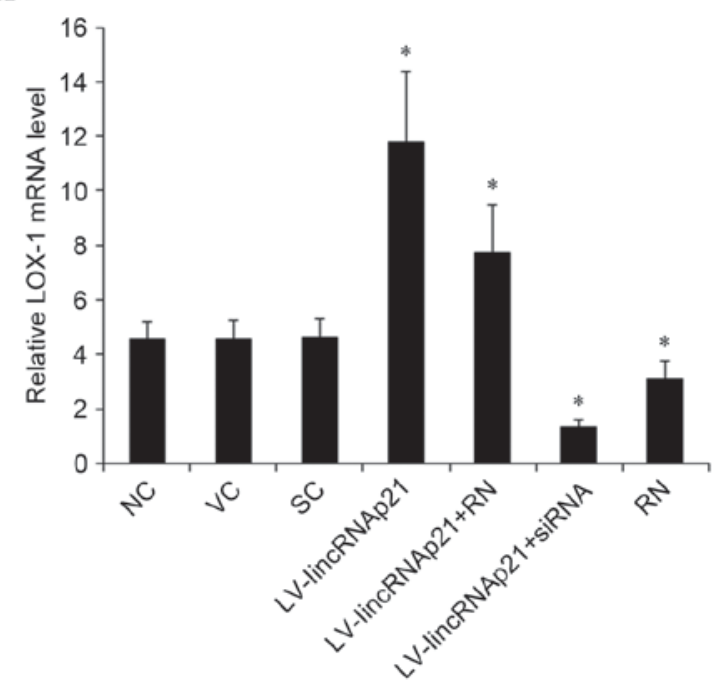

B
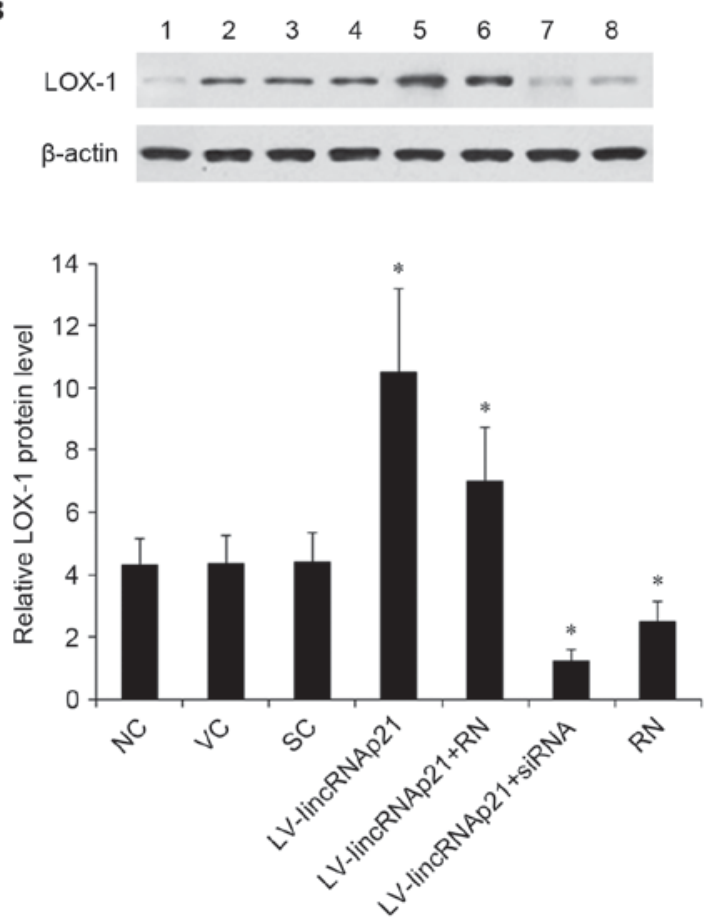

Figure 4. Effect of lincRNA-p21 on expression of LOX-1 in HCAECs under ox-LDL treatment. HCAECs were transduced with LV-lincRNA-p21 or LV-lincRNA-p21-siRNA to overexpress or knockdown lincRNA-p21, respectively. NC, VC and SC were used as controls. The cells were treated with $90 \mu \mathrm{g} / \mathrm{ml}$ ox-LDL for $48 \mathrm{~h}$ with or without $1 \mu \mathrm{M}$ of selective protein kinase C- $\delta$ inhibitor, RN. (A) The LOX-1 mRNA levels was measured with reverse transcription-quantitative polymerase chain reaction and expressed as fold-changes to that of HCAECs under normal culture conditions $(0 \mu \mathrm{g} / \mathrm{ml}$ ox-LDL treatment) for $24 \mathrm{~h}$ (designated as 1). (B) The LOX-1 protein levels were measured with western blot analyses: Lane 1, HCAECs under normal culture conditions ( $0 \mu \mathrm{g} / \mathrm{ml}$ ox-LDL treatment) for $24 \mathrm{~h}$; lanes $2-8$, HCAECs treated with $90 \mu \mathrm{g} / \mathrm{ml}$ ox-LDL for $48 \mathrm{~h}$; and plus, lane 2, NC; lane 3, VC; lane 4, SC; lane 5, LV-lincRNA-p21; lane 6, LV-lincRNA-p21 + RN; lane 7, lincRNA-p21-siRNA; lane 8, RN. b-actin blotting was used as a loading control. Density of the LOX-1 blot was normalized against that of the $\beta$-actin blot to obtain a relative blot density, which was expressed as fold-changes to that of HCAECs under normal culture conditions $(0 \mu \mathrm{g} / \mathrm{ml}$ ox-LDL treatment $)$ for $24 \mathrm{~h}$ (designated as 1). "P<0.05 vs. NC, VC and SC. HCAECs, human coronary artery endothelial cells; ox-LDL, oxidized low-density lipoprotein; $\mathrm{LV}$, lentivirus; $\mathrm{NC}$, no LV transduction; VC, blank control lentivirus; SC, scramble control lentivirus; lincRNA-p21, long intergenic noncoding RNA p21; RN, Rottlerin; siRNA, small interfering RNA.
A

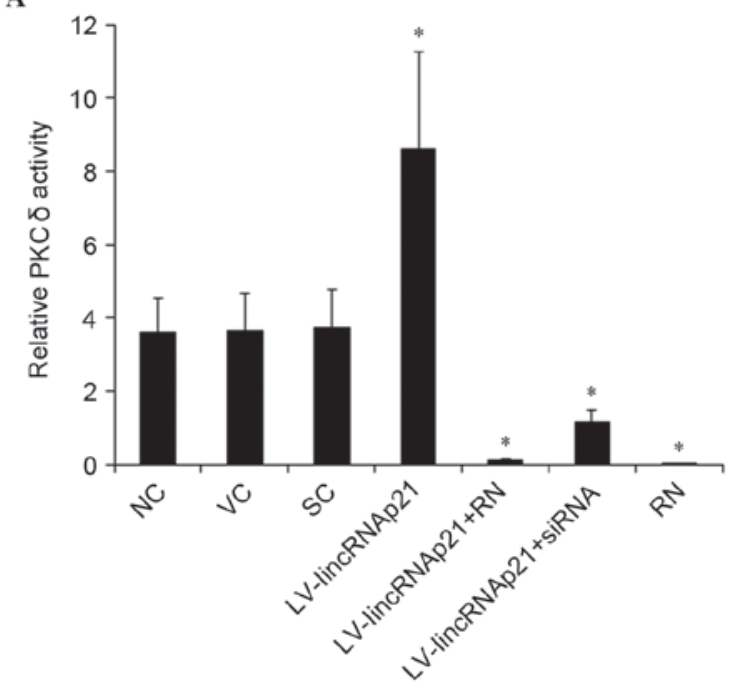

B
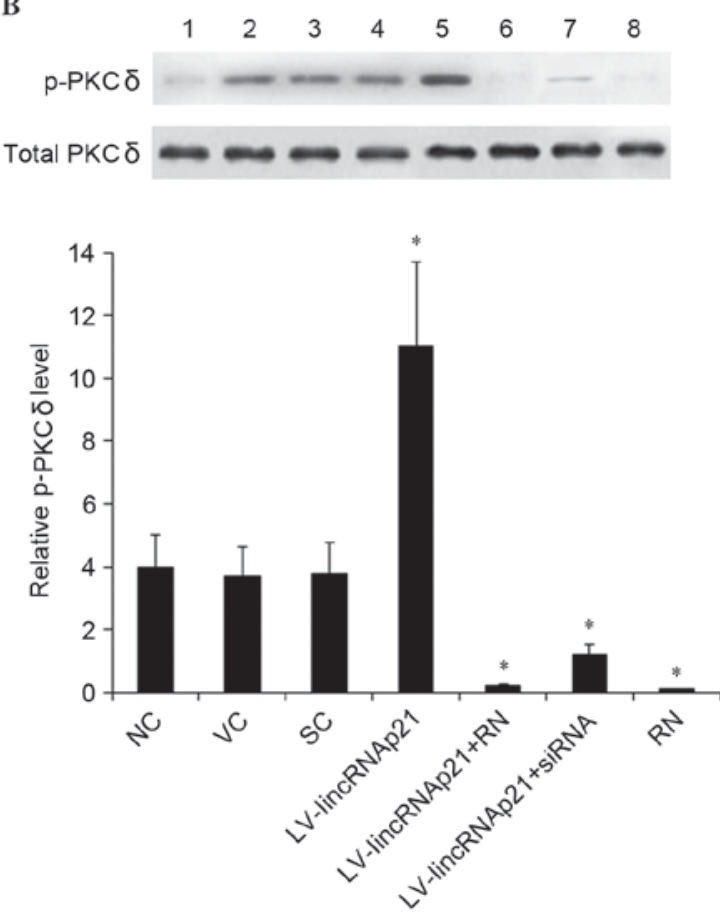

Figure 5. Effect of linkRNA-p21 on PKC $\delta$ activity and p-PKCס levels in in HCAECs under ox-LDL treatment. HCAECs were transduced with LV-lincRNA-p21 or LV-lincRNA-p21-siRNA to overexpress or knockdown lincRNA-p21, respectively. NC, VC and SC were used as controls. The cells were treated with $90 \mu \mathrm{g} / \mathrm{ml}$ ox-LDL for $48 \mathrm{~h}$ with or without $1 \mu \mathrm{M}$ selective PKC $\delta$ inhibitor, RN. (A) The PKC 8 activity was measured and expressed as fold-changes to that of HCAECs under normal culture conditions $(0 \mu \mathrm{g} / \mathrm{ml}$ ox-LDL treatment) for $24 \mathrm{~h}$ (designated as 1) (B) Levels of total PKC 8 and p-PKC $\delta$ at Tyr 155 were determined by western blot analyses: Lane 1, HCAECs under normal culture conditions ( $0 \mu \mathrm{g} / \mathrm{ml}$ ox-LDL treatment) for $24 \mathrm{~h}$; lanes $2-8$, HCAECs treated with $90 \mu \mathrm{g} / \mathrm{ml}$ ox-LDL for $48 \mathrm{~h}$; and plus, lane 2, NC; lane 3, VC; lane 4, SC; lane 5, LV-lincRNA-p21; lane 6, LV-lincRNA-p21 + RN; lane 7, lincRNA-p21-siRNA; lane 8, RN. The total PKCd level was not significantly altered by ox-LDL treatment. Density of the p-PKCD (Tyr 155) blot was normalized against that of total PKC $\delta$ to obtain a relative blot density, which was expressed as fold-changes to that of HCAECs under normal culture conditions $(0 \mu \mathrm{g} / \mathrm{ml}$ ox-LDL treatment) for $24 \mathrm{~h}$ (designated as 1$)$. ${ }^{.} \mathrm{P}<0.05 \mathrm{vs} . \mathrm{NC}, \mathrm{SC}$ and VC. HCAECs, human coronary artery endothelial cells; ox-LDL, oxidized low-density lipoprotein; $\mathrm{PKC} \delta$, protein kinase $\mathrm{C} \delta$; p-, phosphorylated; LV, lentivirus; $\mathrm{NC}$, no LV transduction; VC, blank control lentivirus; SC, scramble control lentivirus; lincRNA-p21, long intergenic noncoding RNA p21; RN, Rottlerin; siRNA, small interfering RNA. 
and/or ii) directly activating p53. As inhibition of PKC $\delta$ activity blocked the majority, but not all, of the enhancing effect of lincRNA-p21 on oxLDL-induced expression of LOX-1 and apoptosis, other mechanisms may be involved. Future studies are required to determine this.

Other than atherosclerosis, LOX-1 is reportedly also critical in the pathogenesis of hypertension, myocardial infarction, congestive heart failure, vascular diseases and thrombosis (7). It will be intriguing to determine the role of oxLDL/LOX-1/lincRNA-p21 signaling in other cardiovascular diseases in addition to atherosclerosis in future studies. In conclusion, the findings of the present study suggest that lincRNA-p21 is a major mediator of oxLDL-induced expression of LOX-1 and apoptosis in human vascular endothelial cells, predominantly by activating PKCס. This provides novel insights into the role of lincRNA-p21 in the pathogenesis of atherosclerosis.

\section{References}

1. Chen Y, Li D, Xu Y, Zhang Y, Tao L, Li S, Jiang Y and Shen X: Essential oils from Fructus A. zerumbet protect human aortic endothelial cells from apoptosis induced by Ox-LDL in vitro Evid Based Complement Alternat Med 2014: 956824, 2014.

2. Hansson GK: Inflammation, atherosclerosis, and coronary artery disease. N Engl J Med 352: 1685-1695, 2005.

3. Wang Y, Zhang Y, Zhu Y and Zhang P: Lipolytic inhibitor G0/G1 switch gene 2 inhibits reactive oxygen species production and apoptosis in endothelial cells. Am J Physiol Cell Physiol 308 C496-C504, 2015

4. Chang HC, Chen TG, Tai YT, Chen TL, Chiu WT and Chen RM: Resveratrol attenuates oxidized LDL-evoked Lox-1 signaling and consequently protects against apoptotic insults to cerebrovascular endothelial cells. J Cereb Blood Flow Metab 31: 842-854, 2011

5. Mitra S, Deshmukh A, Sachdeva R, Lu J and Mehta JL: Oxidized low-density lipoprotein and atherosclerosis implications in antioxidant therapy. Am J Med Sci 342: 135-142, 2011.

6. Ross R: The pathogenesis of atherosclerosis: A perspective for the 1990s. Nature 362: 801-809, 1993.

7. Taye A and El-Sheikh AA: Lectin-like oxidized low-density lipoprotein receptor 1 pathways. Eur J Clin Invest 43: 740-745, 2013.

8. Xu S, Ogura S, Chen J, Little PJ, Moss J and Liu P: LOX-1 in atherosclerosis: Biological functions and pharmacological modifiers. Cell Mol Life Sci 70: 2859-2872, 2013.

9. Pirillo A, Norata GD and Catapano AL: LOX-1, OxLDL, and atherosclerosis angela. Mediators Inflamm 2013: 152786, 2013.

10. Mercer TR and Mattick JS: Structure and function of long noncoding RNAs in epigenetic regulation. Nat Struct Mol Biol 20: 300-307, 2013

11. $\mathrm{Hu}$ W, Alvarez-Dominguez JR and Lodish HF: Regulation of mammalian cell differentiation by long non-coding RNAs. EMBO Rep 13: 971-983, 2012.
12. Song X, Shan D, Chen J and Jing Q: miRNAs and lncRNAs in vascular injury and remodeling. Sci China Life Sci 57: 826-835, 2014.

13. Yang N, Fu Y,Zhang H, Sima H,Zhu N and Yang G: LincRNA-p21 activates endoplasmic reticulum stress and inhibits hepatocellular carcinoma. Oncotarget 6: 28151-28163, 2015.

14. He C, Ding JW, Li S, Wu H, Jiang YR, Yang W, Teng L, Yang J and Yang J: The role of long intergenic noncoding RNA p21 in vascular endothelial cells. DNA Cell Biol 34: 677-683, 2015.

15. Wu G, Cai J, Han Y, Chen J, Huang ZP, Chen C, Cai Y, Huang H, Yang Y, Liu Y, et al: LincRNA-p21 regulates neointima formation, vascular smooth muscle cell proliferation, apoptosis and atherosclerosis by enhancing p53 activity. Circulation 130: 1452-1465, 2014.

16. Mackness MI and Durrington PN: Lipoprotein separation and analysis for clinical studies. In: Lipoprotein Analysis: A Practical Approach. Converse CA and Skinner ER (eds). Oxford University Press, Oxford, pp1-42, 1992.

17. Sparrow CP, Parthasarathy S and Steinberg D: Enzymatic modification of low density lipoprotein by purified lipoxygenase plus phospholipase A2 mimics cell-mediated oxidative modification. J Lipid Res 29: 745-753, 1988.

18. Perissi V, Scafoglio C, Zhang J, Ohgi KA, Rose DW, Glass CK and Rosenfeld MG: TBL1 and TBLR1 phosphorylation on regulated gene promoters overcomes dual CtBP and NCoR/SMRT transcriptional repression checkpoints. Mol Cell 29: 755-766, 2008.

19. Livak KJ and Schmitten TD: Analysis of relative gene expression data using real-time quantitative PCR and the 2(-Delta Delta C(T)) Method. Methods 25: 402-408, 2001.

20. Li X, Pabla N, Wei Q, Dong G, Messing RO, Wang CY and Dong Z: PKC-delta promotes renal tubular cell apoptosis associated with proteinuria. J Am Soc Nephrol 21: 1115-1124, 2010.

21. Konishi H, Tanaka M, Takemura Y, Matsuzaki H, Ono Y, Kikkawa U and Nishizuka Y: Activation of protein kinase $\mathrm{C}$ by tyrosine phosphorylation in response to $\mathrm{H} 2 \mathrm{O} 2$. Proc Natl Acad Sci USA 94: 11233-11237, 1997.

22. Ramnath RD, Sun J, Adhikari S, Zhi L and Bhatia M: Role of PKC-delta on substance P-induced chemokine synthesis in pancreatic acinar cells. Am J Physiol Cell Physiol 294: C683-C692, 2008.

23. Qin B, Cao Y, Yang H, Xiao B and Lu Z: MicroRNA-221/222 regulate ox-LDL-induced endothelial apoptosis via Ets-1/p21 inhibition. Mol Cell Biochem 405: 115-124, 2015.

24. Coutinho I, Pereira C, Pereira G, Gonçalves J, Côrte-Real M and Saraiva L: Distinct regulation of p53-mediated apoptosis by protein kinase $C \alpha, \delta, \varepsilon$ and $\zeta$ : Evidence in yeast for transcription-dependent and -independent p53 apoptotic mechanisms. Exp Cell Res 317: 1147-1158, 2011

25. Wu HM, Schally AV, Cheng JC, Zarandi M, Varga J and Leung PC: Growth hormone-releasing hormone antagonist induces apoptosis of human endometrial cancer cells through pkc $\delta$-mediated activation of p53/p21. Cancer Lett 298: 16-25, 2010.

26. Schisano B, Tripathi G, McGee K, McTernan PG and Ceriello A: Glucose oscillations, more than constant high glucose, induce p53 activation and a metabolic memory in human endothelial cells. Diabetologia 54: 1219-1226, 2011. 